\title{
How to Get Published: Practical Advice
}

\author{
Taco Brandsen ${ }^{1} \cdot$ Susan Appe $^{2} \cdot$ Ruth Simsa $^{3}$
}

Published online: 7 May 2021

(C) International Society for Third-Sector Research 2021

As the number of submissions to Voluntas has expanded, so has the number of rejections. Mostly, these reflect assessments of the quality of the analysis or the underlying data. However, in a number of cases, the rejections are for reasons that are entirely avoidable. In this short editorial, we will suggest some practical advice to getting past the first several hurdles-and perhaps beyond.

\section{Step Outside Yourself-Reflect on Why Others Would Care to Read Your Work}

To publish successfully, it is important to learn to read a text through the eyes of others. Authors, editors and reviewers examine manuscripts from different perspectives. Authors often begin by writing up their project and only afterward consider a choice of journal. Editors and reviewers start from the other end: How does a particular piece of research contribute to the general academic debate on this issue? How does it fit similar texts published in the journal? In other words, it is better to move the argument from the general to the specific, not the other way round, and define from the outset how the findings contribute to the state of the art. A good mental exercise is to try to imagine that readers have little to no interest whatsoever in your project and country (for some people/nationalities, in

Taco Brandsen

t.brandsen@fm.ru.nl

Radboud University, Nijmegen, The Netherlands

2 University at Albany, SUNY, Albany, NY, USA

3 Vienna University of Economics and Business, Vienna, Austria our experience, this tends to be harder than for others). Ask yourself: Why would they read it anyway?

\section{Think About the Reviewers-What Criteria will they use to Assess the Manuscript?}

Once it is out for review, based on our experience reviewers are looking for quality, concision and coherence. In addition, time and time again reviewers comment that submissions fall short on pushing the conversation forward in the field. This is the case across many types of submissions: Whether the submission seeks to fill a research gap, explore a less known phenomenon or further our understanding about what we think we know; in addition, it is a comment by reviewers for both qualitative and quantitative manuscripts, single-case studies and comparative analyses. In part, we would suggest, this comment highlights the burden on authors, as noted, to make a concerted effort to draw explicitly on the literature in the field (in Voluntas and elsewhere) and make sure the findings contribute to the broader state of the art.

Reviewers are asked to evaluate your manuscript using several general criteria standard to social science research: strengths and weaknesses of the theoretical and, if applicable, methodological approaches of the manuscript, a topic pertinent to the field, proper references, etc. Reviewers for Voluntas are also asked two additional questions, written here verbatim:

1. Given the international readership of Voluntas, does this paper have relevance outside its country of origin?

2. Given that Voluntas is an interdisciplinary journal, is this paper accessible to readers from a variety of disciplines? 
Keeping these questions in mind as you develop your manuscript will be extremely helpful. They help prompt you to define your terms and do so taking into account Voluntas' intended audience. Such an approach allows you to frame the text effectively from the beginning of the writing stage and therefore tell a story relevant well beyond the context about which you are writing.

\section{Find Common Ground-Trace the History of the Discussion in the Journal}

A related point: It is good to check whether others have published on similar topics in the journal. That is, you can find certain trends of publication in journals (e.g., see Calcagno, Demoinet, Gollner, Guidi, Ruths \& de Mazancourt (2012) for the physical sciences; Martin (2014) for the social sciences). If the journal has not published on the topic of your manuscript, that begs the question whether it is the right journal. If it has, these articles present an easy starting point for demonstrating that the article is relevant and timely. It is not compulsory, but practical, to reference some of these previous publications. After all, reviewers often include scholars who have previously published in the journal.

This also allows for what Martin (2014) calls a "a common ground or an encompassing debate" to be constructed by using related and common language for scholarly interaction among authors and the readership of Voluntas. Two of us recently identified patterns over the last five years in Voluntas. We observed an increased number of submissions on the topics of social enterprise, philanthropy, grassroots activities and social movements. Some of the classical topics discussed in Voluntas remain, but indeed have seen changes. For example, submissions about volunteering have a greater focus on non-conventional, flexible forms of volunteering, and at least for now, we recognize a relative decline of classical management research on nonprofit organizations (Simsa \& Brandsen, 2020). These trends might inform how you frame your research and contributions to the field, or alternatively even allow you to present topics that are currently missing from observed patterns but that deserve attention.

\section{Treat the Abstract as More than a Formality}

The short summary required by journals often seems to be written in a rush. However, it is a crucial piece of the article and requires serious thought. Keep in mind that the abstract gives readers the very first impression of your work and might prime their attitude toward it. While somewhat anecdotal, we find that the quality of the abstract is related to the quality of the manuscript itself. The abstract serves as a very brief overview of your research. It tells the reader (e.g., the editors and reviewers) what you did, why you did it, how you did it, what you found, and what it means. The abstract should essentially be a "mini" article. You should briefly state the purpose and the problem/puzzle of the research (introduction/literature review), how the problem was studied (methods), the principal findings (results), and what the findings mean (discussion and conclusion). However, the abstract should be understandable without readers knowing the whole text, avoid abbreviations and very specific arguments that are only explained later!

It is important to be clear and straightforward. Like the manuscript itself, the abstract should consider (1) the journal's specific audience and (2) the main point you are making in your manuscript. It needs to convey your text's main contribution, aims and conclusion to the editor, the reviewers and readers. And, again like the manuscript itself, the abstract must speak beyond the specific case you are analyzing in your article. Because it is the first impression on readers, it helps them to decide if the article deserves to be read. Additionally, you should pay attention to keywords so that your article can be found through online search engines once it is published.

\section{Meet the Minimum Requirements and Escape a Desk Reject}

Manuscripts themselves are uploaded into the system and initially screened for adherence to the submission guidelines. This includes limited word count (for Voluntas maximum words count is 8,000 , inclusive of everything) and various disclosures about, for examples, conflict of interest and funding sources. Once it meets the submission requirements, the editors provide a desk review. This is when we do an initial review of the Voluntas criteria (does it speak to international and interdisciplinary audiences?), and consider more general social science standards such as a theoretical framework, methodological approach, analysis and contributions. Once we decide it meets the standard to go out for review, we select reviewers based on our own knowledge of colleagues who are well-positioned to review the submission, given the topic, empirical setting and/or methodology. We also consider how the authors classified the submission according to the pre-defined classifications in the manuscript system. We recommend that authors take these classifications seriously, as it provides a further indication of who might be a qualified and appropriate reviewer. 


\section{Take Reviewers Seriously-Never Show Your True Feelings}

Getting to the revision stage is already a major achievement. After this, the majority of manuscripts end up being published. But, should you wish to fail at this advanced stage, the best way to do it is to demonstrate that you refuse to take reviewers seriously. This can be done by making minimal, token changes to the text, hoping that the reviewers are careless enough not to notice. Another good strategy to get rejected is to show anger or annoyance, by implying that reviewers have not understood the text or read it badly: "as we already explained on page $9, \ldots$ ". "The reviewer has misunderstood this point." Everyone has the experience of disliking a reviewer passionately, but showing it is simply not helpful.

Should you wish to get published, write a point-by-point response to the reviewers' comments and stay gracious. There is no need to follow reviewers' advice slavishly: Sometimes comments are contradictory or impossible to address. In that case, please explain why you have chosen to ignore the comment. On the whole, though, try to do them justice.

\section{Further Resources}

Other resources that might help you think about how to write a quality manuscript exist. For example, the Academy of Management Journal in 2011 had a seven-part series called "Publishing in AMJ" that provides advice and strategies for submitting publishable articles to the journal. While speaking to management scholars, many of the suggestions are applicable to the social sciences more generally. Other fields related to third sector studies also have provided resources-about how to publish (Blair et al., 2020) and how to accept rejection (Martin, 2014); and others even critically question the current standards for publication (e.g., Healy, 2017; Tourish, 2020). René Bekkers' "How to Review a Paper" (2020) provides suggestions to reviewers (its intended audience), but in doing so allows authors to think about how their work is likely to be reviewed. The short guide paper is targeted for the review of hypothesis-testing papers, but for those authors who are testing hypotheses, it might provide key considerations to think about.

In sum, academic publishing is a special world, where rules and practices can be tedious. Yet, in our experience, manuscripts do usually improve during the review process. As a result, they are able to contribute more fully to knowledge production and have more of an impact. In the end, it is worth the trouble.

\section{References}

Bekkers, R. (2020). How to review a paper. OSF Preprints. May, 2. Blair, A., Buckley, F., Rashkova, E., \& Stockemer, D. (2020). Publishing in political science journals. European Political Science, 19, 641-652

Calcagno, V., Demoinet, E., Gollner, K., Guidi, L., Ruths, D., \& de Mazancourt, C. (2012). Flows of research manuscripts among scientific journals reveal hidden submission patterns. Science, $338,1065-1069$

Healy, K. (2017). Fuck Nuance. Sociology Theory, 35(2), 118-127

Martin, E. (2014). How to write a good article. Current Sociology, 62(7), 949-955

Simsa, R., \& Brandsen, T. (2020). The evolution of third sector research and the journal voluntas: The editors' impressions. VOLUNTAS: International Journal of Voluntary and Nonprofit Organizations, 32(1), 1-2

Tourish, D. (2020). The triumph of nonsense in management studies. Academy of Management Learning \& Education, 19(1), 99-109

Publisher's Note Springer Nature remains neutral with regard to jurisdictional claims in published maps and institutional affiliations. 